\title{
The eyes fixate the optimal viewing position of task-irrelevant words
}

\author{
Daniel Smilek, Grayden J. F. Solman, \\ Peter Murawski, and Jonathan S. A. Carriere \\ University of Waterloo, Waterloo, Ontario, Canada
}

\begin{abstract}
We evaluated whether one's eyes tend to fixate the optimal viewing position (OVP) of words even when the words are task irrelevant and should be ignored. Participants completed the standard Stroop task, in which they named the physical color of congruent and incongruent color words without regard to the meanings of the color words. We monitored the horizontal position of the first eye fixation that occurred after the onset of each color word to evaluate whether these fixations would be at the OVP, which is just to the left of word midline. The results showed that (1) the peak of the distribution of eye fixation positions was to the left of the midline of the color words, (2) the majority of the fixations landed on the left side of the color words, and (3) the average leftward displacement of the first fixation from word midline was greater for longer color words. These results suggest that the eyes tend to fixate the OVP of words even when those words are task irrelevant.
\end{abstract}

When an individual intentionally reads a word, the eyes tend to land on a specific location on the word, known as the optimal viewing position (OVP; Dunn-Rankin, 1978; O’Regan, 1981; Rayner, 1979). The OVP is just to the left of the center of the word, and the available data suggest that it tends to be farther left of center for longer words (O’Regan, Lévy-Schoen, Pynte, \& Brugaillère, 1984). When the eyes fail to land on the OVP, intentional reading is slowed by approximately $20 \mathrm{msec}$ for each deviation in letter position from the OVP (O'Regan \& Jacobs, 1992). Here, we evaluated whether the eyes are biased to fixate the OVP even when the word is irrelevant to the task at hand and the demands of the task require lexical processing of the word to be suppressed.

To address this issue, we monitored eye position while participants completed the standard Stroop task (Stroop, 1935). In the Stroop task, participants are shown a color word (e.g., red) in a physical color that is either congruent (e.g., red) or incongruent (e.g., blue) with the color word. Participants are required to suppress lexical processing of the color word and to name the physical color. The standard result is that participants are much slower to name the physical color when that color is incongruent than when it is congruent with the meaning of the color word (see MacLeod, 1991, for a review). This result is often taken to indicate that the meaning of the color word is processed even though it is irrelevant to the task. We reasoned that if the oculomotor system is engaged by these task-irrelevant words, the eyes might fixate to the left of center (the OVP) of the colored words, even though participants are trying to suppress lexical processing of the color word and to name the physical color instead.
There are several good reasons to believe that the eyes might fixate the OVP of words even when the words are task irrelevant. One reason is that word reading is a highly practiced task. As such, it seems reasonable that the stereotypic eye movements associated with intentional reading might generalize to situations in which word reading is detrimental to task performance. Another reason is suggested by recent studies of single-letter coloring in the Stroop task. When a single letter of a Stroop word is colored, the Stroop effect is radically reduced, and sometimes completely eliminated (e.g., Besner, Stolz, \& Boutilier, 1997; Manwell, Roberts, \& Besner, 2004). In a recent report, Parris, Sharma, and Weekes (2007) extended these findings by examining how the magnitude of the Stroop effect varied as a function of the position of the letter that was colored. In one of their studies, Parris et al. colored the initial letter, the final letter, the letter to the right of center, or - critically - the letter just to the left of center, which approximated the OVP. The Stroop effect observed in these conditions was also compared with the effect observed in a condition in which all of the letters were colored, as in the standard Stroop task. As expected, when all of the letters of the word were colored, the results showed a substantial Stroop effect. More important, the Stroop effect was substantially diminished when a single letter was colored at each of the letter positions tested, except when the letter to the left of center (at the OVP) was colored. When only the letter at the OVP for that word was colored, the Stroop effect was similar in size to the Stroop effect observed when all letters were colored.

Further evidence that fixating the OVP is an important moderator of Stroop performance comes from the second 
experiment reported by Parris et al. (2007). In this experiment, they again colored only a single letter in Stroop words in such a way that the initial letter, the last letter, or the letter at the OVP was colored. Importantly, in this experiment, the word was shifted horizontally from trial to trial in such a way that the colored letter always appeared in the same location as the preceding central fixation cue. Presenting the colored letter at fixation was meant to increase the likelihood that participants fixated the colored letter, and thus the OVP, when the letter at the OVP was colored. The results again showed that the Stroop effect was reduced when a single letter was colored at each of the letter positions except the OVP. Thus, fixating the OVP seems to eliminate the Stroop dilution caused by singleletter coloring. Given the important role of the OVP in this task, it seems likely that the eyes might be biased toward fixating the OVP of a word even when that word is task irrelevant, as in the standard Stroop task. To our knowledge, however, this possibility has not been directly explored.

A demonstration of an oculomotor bias to fixate the OVP in the Stroop task would have important implications for the extensive literature concerned with understanding Stroop task performance. Studies of Stroop performance have largely focused on the cognitive processes involved (e.g., semantic activation, automaticity of reading, and color naming) and have not considered the possible involvement of the oculomotor system (see Jensen \& Rohwer, 1996, and MacLeod, 1991, for reviews). If an oculomotor bias is present, it would be important to consider oculomotor involvement as an additional constraint on theories of Stroop performance. As well, an oculomotor bias in the Stroop task would point toward possible oculomotor explanations of several dilutions of the Stroop effect (e.g., Besner et al., 1997; Manwell et al., 2004). That is, since word reading is slowed when the eyes do not fixate the OVP, Stroop dilutions may occur because the eyes are prevented from fixating - or are drawn away from - the OVP of a color word.

We evaluated whether the eyes are biased to fixate the OVP of Stroop words by measuring the horizontal position of the first distinct eye fixation that occurred after the onset of the color word. We expected that an oculomotor bias toward the OVP would be manifested in several different ways in the Stroop task. First, overall eye fixations should be normally distributed, with the peak of the distribution falling to the left of center of each color word. Second, we expected that the majority of first fixations on color words would fall on the left side of those words. Finally, because the OVP is farther left from midline as word length increases (O'Regan et al., 1984), we expected to find the average landing position of the first fixation to also be farther left from word midline as word length increased. To confirm the latter expectation, we included the color words red, blue, green, and yellow, which contain three, four, five, and six letters, respectively.

\section{METHOD}

Thirteen undergraduate students ( 3 male, 10 female) with normal or corrected-to-normal vision from the University of Waterloo participated in a 1-h session for course credit. One participant was excluded from analysis due to poor eyetracking data.

The stimuli consisted of the color words red, blue, green, and yellow. In each trial, one word was presented at the center of the screen in a lowercase sans serif font with one space between each letter. A fixation cross was not presented, to prevent the eyes from being biased toward a specific location on the screen. Each letter subtended on average $1.0^{\circ}$ horizontally (from $0.2^{\circ}$ for the letter "l" to $1.7^{\circ}$ for "w") and $1.4^{\circ}$ vertically (from $1.2^{\circ}$ for " $\mathrm{w}$ " to $1.6^{\circ}$ for " $\mathrm{g}$ "). Words subtended $7.7^{\circ}$ horizontally on average, ranging from $4.6^{\circ}$ for red to $10.8^{\circ}$ for yellow. Using this large letter size allowed us to maximize our ability to detect eye movements to the OVP of shorter color words such as red and blue, for which the OVP is very close to the center of the word. We assumed that increasing the size of the Stroop words would not influence the processing of these words or the magnitude of the Stroop effect, but that it would magnify eye movements made to the OVP. All stimuli were colored using colors from the set of color words: red (RGB: 255, 0, 0), blue (RGB: 0, 0 , 255), green (RGB: 0, 255, 0), and yellow (RGB: 255, 255, 0).

The stimuli were displayed and responses collected using SR Research Experiment Builder software (Version 1.4.128 RC) on a Dell Latitude D800 notebook computer. This was connected to an SR Research EyeLink 1000 eyetracking device. Stimuli were presented on a ViewSonic G225f 21 -in. CRT monitor (20.0-in. viewable area) at a resolution of $1,280 \times 1,024$ pixels, with participants viewing from a chinrest approximately $63.5 \mathrm{~cm}$ away. Verbal response time (RT) was measured with a handheld microphone connected to a Psychology Software Tools serial response box.

Participants were instructed to verbally report the color of each word that appeared on screen as quickly and accurately as possible. Each trial began with a blank screen presented for $525 \mathrm{msec}$, followed by one of the four color words presented in the center of the screen. The word was presented until response, at which time the screen went blank while the researcher coded the trial for accuracy. Trials for which the participant made an incorrect response or for which the voice key was inadvertently triggered were repeated later at a random time. Participants completed 12 practice trials and a minimum of 120 experimental trials (assuming no replacements), consisting of 30 trials for each color word, half congruent and half incongruent.

\section{RESULTS}

The standard Stroop effect was observed in both RTs and errors, whereby RTs were slower and errors were greater on incongruent $\left(M_{\mathrm{RT}}=752 \mathrm{msec}, M_{\text {error }}=7.4 \%\right)$ than on congruent $\left(M_{\mathrm{RT}}=620 \mathrm{msec}, M_{\text {error }}=2.1 \%\right)$ trials [RTs, $t(11)=9.87, p<.001$; errors, $t(11)=3.76$, $p<.005]$. The overall magnitude of the Stroop effect $(132 \mathrm{msec})$ was similar to that in other Stroop studies that measured a vocal response (e.g., $105 \mathrm{msec}$ when all letters were colored, as found by Parris et al., 2007). This result suggests that the large letter size that we used for the color words in the present study did not produce Stroop effects substantially different from those typically found with smaller letters. This finding supports our assumption that increasing the size of the Stroop words does not substantially influence the participants' processing of those words.

More important, we found that the first eye fixations after onset of the color words were systematically biased to fixate the OVP. This bias is clearly seen in Figure 1A, which shows the proportion of first fixations that landed at various horizontal positions for each of the color words. The zero on the $x$-axis indicates the midline of each color word, negative numbers indicate distance leftward from 


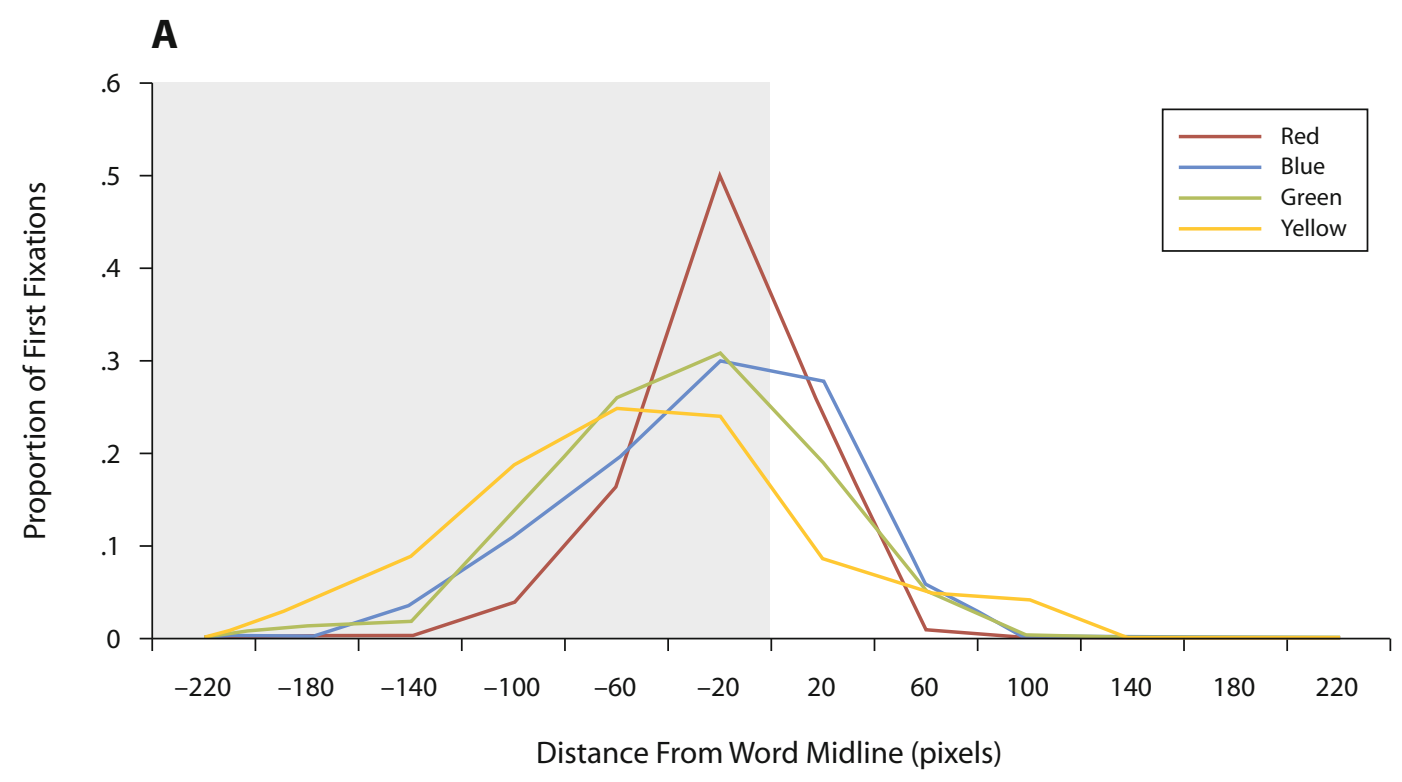

B
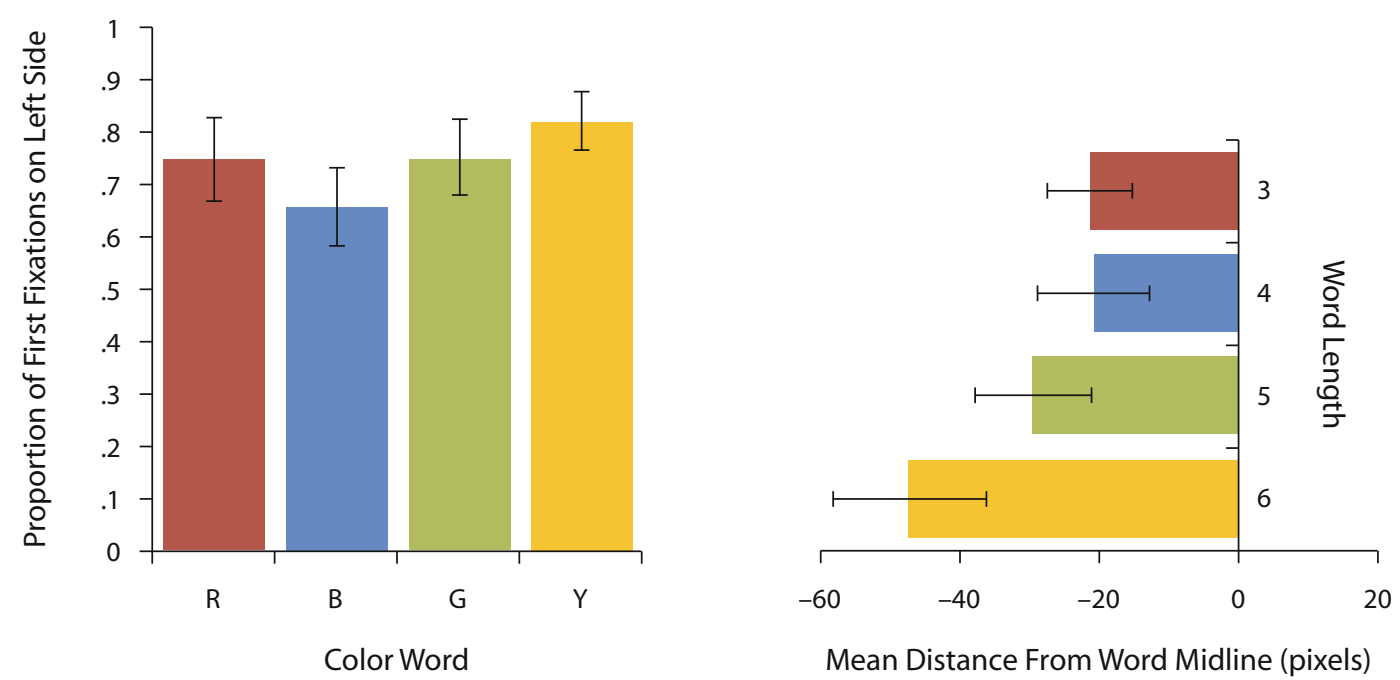

Figure 1. (A) Proportion of first fixations on each color word as a function of position from the center of the word in 40-pixel (half-letter) bins (negative values correspond to the left side of the word). (B) Proportion of first fixations landing on the left side of each color word (R, red; B, blue; G, green; Y, yellow). Error bars depict one standard error of the mean. (C) Mean distance (in pixels) from midline of the first fixation after onset of each color word (negative values correspond to the left side of word midline). Error bars depict one standard error of the mean.

word midline, and positive numbers indicate distance rightward from word midline. Note that the distributions are roughly normal and that the peaks of the distributions are on the left side of the color words, which is the general location of the OVP.

A further confirmation of this pattern is illustrated in Figure 1B, which shows the proportion of first fixations after onset of the color words that landed on the left side of those words. As can be seen in the figure, the majority of the first fixations landed on the left side of the color words. This was statistically verified by a series of onetailed, single-sample $t$ tests comparing the probability of fixating the left side of the words against a value of .5 (chance) $[$ red, $t(11)=2.74, p<.01$; blue, $t(11)=1.72$, $p=.057$; green, $t(11)=3.08, p<.006$; and yellow, $t(11)=5.19, p<.001]$.

Finally, Figure 1C shows that first-fixation position depended on word length. The figure depicts the average distance from word midline (in pixels) of the first eye fixations as a function of color word length. The negative numbers on the $x$-axis again correspond to positions leftward of word midline. Inspection of the figure clearly shows that the average leftward displacement of the first fixation increased as word length increased $[F(3,33)=$ 
9.35, $\left.M S_{\mathrm{e}}=196.5, p<.001\right]$. That is, the average horizontal displacement of the first fixation from midline was the greatest for the longest color word (yellow) and the least for the shortest color words (red and blue). This finding shows that the position of the first fixation after word onset is governed by the length of the word and does not simply reflect a general bias to fixate left of center. This finding is also consistent with the results reported by O'Regan et al. (1984), which showed that the OVP is farther leftward from word midline as word length is increased.

\section{DISCUSSION}

The present findings clearly indicate that one's eyes reliably fixate the OVP of a word, even when that word is irrelevant to the task at hand and doing so may be detrimental to task performance. The finding that the horizontal position of the first fixation after onset of the color word depends on word length is particularly striking and provides strong evidence that the bias in eye movements is driven by the task-irrelevant color words. The present findings extend previous demonstrations that the eyes tend to fixate the OVP of words during intentional word reading (Dunn-Rankin, 1978; O'Regan, 1981; Rayner, 1979).

The present results also have important implications for studies and theories of performance in the Stroop task. Our findings imply that the "automatic" or involuntary aspect of word reading in the Stroop task includes not only the purely cognitive processes involved in reading (e.g., lexical and semantic access) but also early oculomotor accommodation of the color word. This early accommodation is likely based on visual properties of the word (e.g., word length, letter spacing) and occurs very early in word processing (see Ducrot \& Pynte, 2002). In future studies, it will be interesting to evaluate the extent to which this oculomotor accommodation can be dissociated from cognitive contributions to the standard Stroop effect.

The present findings have particular relevance for studies concerned with modulations of the magnitude of the Stroop effect (e.g., Besner et al., 1997; Kahneman \& Chajczyk, 1983; Manwell et al., 2004; Parris et al., 2007). For instance, consider the Stroop dilutions demonstrated by Besner and colleagues (Besner et al., 1997; Manwell et al., 2004) whereby the magnitude of the Stroop effect is reduced when only a single letter in the color word is colored and spatially cued. The explanation for these findings was that both the letter color and the spatial cue allow easier separation of the information from the color word and the physical color, resulting in a reduction of interference between the two sources. In contrast to this explanation, the present results suggest the possibility that coloring and spatially cuing a single letter actually directs overt eye movements away from the OVP on many of the trials, since each letter in the color words was cued and colored equally often across trials. Because fixating away from the OVP is known to slow word reading (i.e., to increase lexical decision times; O'Regan \& Jacobs, 1992), moving the eyes away from the OVP by cuing or single-letter coloring could slow word processing enough to prevent it from interfering with color naming. Unfortunately, in the present data we were unable to compare the magnitude of the Stroop effect across different letter positions, because too few fixations fell far enough away from the OVP to make any comparisons meaningful. Consistent with our explanation, however, Parris et al. found that coloring a single letter reduced the magnitude of the Stroop effect at every letter position except when the colored letter was at the OVP. If our speculation is correct, we would expect that coloring and spatially cuing a single letter would direct overt spatial attention away from the OVP and to the cued and colored letter. More generally, our results raise the possibility that at least some modulation of the Stroop effect might result not only from a direct modulation of purely cognitive processes (e.g., lexical or semantic activation), but also from a modulation of early oculomotor accommodation of the Stroop words.

There are several ways in which our experiment differed from the majority of previous Stroop studies. One difference was that the size of the letters of the Stroop words that we used was greater $\left(1.0^{\circ}\right.$ wide on average $)$ than is typically used in other Stroop studies reported in the literature (e.g., approximately $0.4^{\circ}$ wide on average, in a study by Manwell et al., 2004). Using larger letters allowed us to discriminate between eye positions at the OVP and those at word midline, even in color words with very few letters, for which the OVP is likely very close to word midline (e.g., red and blue). Although there remains the possibility that visually larger Stroop words might be processed differently than smaller words, the fact that we found a Stroop effect similar in magnitude to those obtained in other studies suggests that this is unlikely. Another difference is that we did not present a fixation point before the onset of each color word. We chose to exclude the fixation point so that we would not constrain fixation at the beginning of each trial and unnecessarily bias where participants fixated prior to the onset of each word. As with the change in letter size, we do not believe that this choice substantially limits the generality of our findings.

We will conclude by highlighting that the present findings build on the growing body of evidence showing how cognitive processing must be considered in the context of the physical constraints of the human body (e.g., Clark, 1999; Findlay \& Gilchrist, 2003). The importance of oculomotor behavior has been examined in a broad range of contexts, including visual orienting, reading, and visual search (see Findlay \& Gilchrist, 2003, for a review), and it has been demonstrated in all of these cases that the perceptual constraints imposed by the physical relations between the eyes and a stimulus play a critical role in moderating consequent cognitive processes.

\section{AUTHOR NOTE}

This work was supported by NSERC with a grant to D.S. and a graduate scholarship to J.S.A.C. We thank Derek Besner for his helpful comments and suggestions. Correspondence concerning this article should be addressed to D. Smilek, Department of Psychology, University of Waterloo, 200 University Ave. West, Waterloo, ON, N2L 3G1 Canada (e-mail: dsmilek@uwaterloo.ca). 


\section{REFERENCES}

Besner, D., Stolz, J. A., \& Boutilier, C. (1997). The Stroop effect and the myth of automaticity. Psychonomic Bulletin \& Review, 4, 221-225.

Clark, A. (1999). An embodied cognitive science? Trends in Cognitive Sciences, 3, 345-351. doi:10.1016/S1364-6613(99)01361-3

Ducrot, S., \& Pynte, J. (2002). What determines the eyes' landing position in words? Perception \& Psychophysics, 64, 1130-1144.

Dunn-RAnkin, P. (1978). The visual characteristics of words. Scientific American, 238, 122-130.

Findlay, J. M., \& Gilchrist, I. D. (2003). Active vision: The psychology of looking and seeing. Oxford: Oxford University Press.

Jensen, A. R., \& Rohwer, W. D., JR. (1966). The Stroop color-word test: A review. Acta Psychologica, 25, 36-93. doi:10.1016/0001 $-6918(66) 90004-7$

Kahneman, D., \& ChaJCZyK, D. (1983). Tests of the automaticity of reading: Dilution of Stroop effects by color-irrelevant stimuli. Journal of Experimental Psychology: Human Perception \& Performance, 9, 497-509. doi:10.1037/0096-1523.9.4.497

MacLEOD, C. M. (1991). Half a century of research on the Stroop effect: An integrative review. Psychological Bulletin, 109, 163-203. doi:10.1037/0033-2909.109.2.163

Manwell, L. A., Roberts, M. A., \& Besner, D. (2004). Single letter coloring and spatial cuing eliminates a semantic contribution to the Stroop effect. Psychonomic Bulletin \& Review, 11, 458-462.

O'REgAN, J. K. (1981). The convenient viewing position hypothesis. In D. F. Fisher, R. A. Monty, \& J. W. Senders (Eds.), Eye movements: Cognition and visual perception (pp. 289-298). Hillsdale, NJ: Erlbaum.

O'Regan, J. K., \& JACOBS, A. M. (1992). Optimal viewing position effect in word recognition: A challenge to current theory. Journal of Experimental Psychology: Human Perception \& Performance, 18, 185-197. doi:10.1037/0096-1523.18.1.185

O'Regan, J. K., Lévy-Schoen, A., Pynte, J., \& Brugaillère, B. (1984). Convenient fixation location within isolated words of different length and structure. Journal of Experimental Psychology: Human Perception \& Performance, 10, 250-257. doi:10.1037/0096-1523.10.2.250

Parris, B. A., Sharma, D., \& Weekes, B. [S.] (2007). An optimal viewing position effect in the Stroop task when only one letter is the color carrier. Experimental Psychology, 54, 273-280. doi:10.1027/1618 $-3169.54 .4 .273$

RAYNER, K. (1979). Eye guidance in reading: Fixation locations within words. Perception, 8, 21-30. doi:10.1068/p080021

STRoop, J. R. (1935). Studies of interference in serial verbal reactions. Journal of Experimental Psychology, 18, 643-662. doi:10.1037/h0054651

(Manuscript received March 10, 2008; revision accepted for publication July 18, 2008.) 